\title{
Integração entre BIM e simulação termoenergética para obtenção de ENCE de uma edificação do exército brasileiro
}

No Brasil, o Programa Nacional de Eficiência Energética em Edificações promove, juntamente com o INMETRO, a avaliação de eficiência energética das edificações por meio da Etiqueta Nacional de Conservação de Energia (ENCE). O regulamento que define os métodos para avaliação energética, no âmbito das edificações públicas, está sendo atualizado, passando a ser denominado Instrução Normativa INMETRO para Classe de Eficiência Energética de Edificações Comerciais, de Serviços e Públicas (INI-C). Para atender Atos Normativos do Governo Federal, os projetos de obras do Exército Brasileiro devem obter ENCE Nível 'A e devem ser elaborados utilizando a metodologia BIM. Na INI-C, há um método que utiliza simulação termoenergética de edificações para sua classificação. O objetivo desse trabalho, portanto, é avaliar a possibilidade de fazer interoperabilidade entre o projeto elaborado em Revit e os softwares utilizados para simulação, indicando estratégias a serem seguidas nesse processo. Para isso, foi realizada uma análise energética de um pavilhão militar pelo método de simulação da INI-C, utilizando três combinações de softwares: modelagem da edificação no ScketchUp e no OpenStudio, caracterização e simulação no EnergyPlus; modelagem, caracterização e simulação da edificação no DesignBuilder; e utilização do projeto em Revit para caracterização e simulação no DesignBuilder, após processo de interoperabilidade. O consumo energético anual simulado na condição real das três modelagens foi, em média, 32.243,44 kWh com desvio máximo de 1,00\% entre as modelagens. Na condição de referência, $80.118,71 \mathrm{kWh}$ com desvio máximo de $0,01 \%$. Em todas, obteve-se ENCE Geral Classe 'A', com média de 59,76\% de economia energética. A modelagem que utiliza interoperabilidade entre Revit e DesignBuilder foi mais vantajosa, com economia de tempo no processo de avaliação e excelente precisão (desvio máximo de 1,00\% em relação às demais). Para usufruir desse potencial, há de se seguir algumas recomendações no processo de interoperabilidade, apresentadas nesse trabalho.

Palavras-chave: Eficiência energética; INI-C; RTQ-C; EnergyPlus; DesignBuilder.

\section{Integration between BIM and thermoenergetic simulation to obtain ENCE from a building of the Brazilian army}

\begin{abstract}
In Brazil, the National Program for Energy Efficiency in Buildings promotes, together with INMETRO, the evaluation of energy efficiency of buildings using the National Energy Conservation Label (ENCE). The regulation that defines the methods for energy evaluation, in the scope of public buildings, is being updated, becoming known as INMETRO Normative Instruction for the Energy Efficiency Class of Commercial, Service and Public Buildings (INI-C). In order to comply with Federal Government Regulatory Acts, the projects of Brazilian Army buildings must obtain ENCE Level ' $A$ ' and must be prepared using the BIM methodology. In INI-C, there is a method that uses thermo-energetic simulation of buildings for their classification. Therefore, the objective of this work is to evaluate the possibility of making interoperability between a project developed in Revit and a software used for simulation, indicating strategies to be followed in this process. For this, an energy analysis of a military pavilion was carried out using the INI-C simulation method, using three combinations of softwares: building modeling in ScketchUp and OpenStudio, characterization and simulation in EnergyPlus; building modeling, characterization and simulation in DesignBuilder; and use of the Revit project for characterization and simulation in DesignBuilder, after an interoperability process. The simulated annual energy consumption in the real condition of the three models was, on average, $32,243.44 \mathrm{kWh}$ with a maximum deviation of $1.00 \%$ between the models. In the reference condition, $80,118.71 \mathrm{kWh}$ with a maximum deviation of $0.01 \%$. In all, General ENCE Class ' $\mathrm{A}$ ' was obtained, with an average of $59.76 \%$ of energy savings. The modeling that uses interoperability between Revit and DesignBuilder was more advantageous, with time savings in the evaluation process and excellent precision (maximum deviation of $100 \%$ in relation to the others). To take advantage of this potential, some recommendations in the interoperability process, presented in this work, must be followed.
\end{abstract}

Keywords: Energy Efficiency; INI-C; RTQ-C; EnergyPlus; DesignBuilder

Topic: Construções e Arquitetura Sustentáveis

Reviewed anonymously in the process of blind peer.
Received: 02/01/2021

Approved: 27/01/2021
Breno Pontes Pimentel (iD

Universidade Federal de Mato Grosso do Sul, Brasil

http://lattes.cnpq.br/4139930229528889

http://orcid.org/0000-0002-3004-6574

brenopontespimentel@gmail.com

Andréa Teresa Riccio Barbosa (iD

Universidade Federal de Mato Grosso do Sul, Brasil

http://lattes.cnpq.br/4025767578391844

http://orcid.org/0000-0002-2021-1310

andrea.barbosa@ufms.br

Marcelo de Miranda Reis (iD)

Instituto Militar de Engenharia, Brasil

http://lattes.cnpq.br/0498662991287399

http://orcid.org/0000-0003-3875-9189

marceloreis@ime.eb.br
Referencing this:

PIMENTEL, B. P.; BARBOSA, A. T. R.; REIS, M. M.. Integração entre BIM e simulação termoenergética para obtenção de ENCE de uma edificação do exército brasileiro. Revista Ibero Americana de Ciências Ambientais, v.12, n.1, p.232-248, 2021. DOI:

http://doi.org/10.6008/CBPC2179-6858.2021.001.0020 


\section{INTRODUÇÃO}

Nos últimos anos, em todo o mundo, tem crescido o consumo energético, principalmente no setor de edificações. Em 2019, as edificações representaram 36\% do consumo energético total no mundo, com tendências crescentes (IEA, 2019a; 2019b).

Segundo Fossati et al. (2016), uma maneira de reduzir o consumo energético dos edifícios é o estabelecimento de políticas de regulação, de avaliação e de classificação do desempenho energético desses. Evans et al. (2009) afirmam que os códigos energéticos de edifícios podem garantir que o consumo de energia possa ser reduzido em até $50 \%$, quando comparados a edifícios que não aplicam técnicas de eficiência energética, e ainda com aumento no conforto térmico dos usuários e com economia de recursos financeiros.

Neste sentido, desde 2014 há no Brasil uma Instrução Normativa do Ministério do Planejamento, Orçamento e Gestão-MPOG dispondo que tanto os projetos quanto as edificações públicas federais, novas ou que recebam retrofit, devem obter a Etiqueta Nacional de Conservação de Energia (ENCE) nível 'A' (MPOG, 2014). Os métodos para avaliação energética desse tipo de edificação são previstos no Regulamento Técnico da Qualidade para o Nível de Eficiência Energética de Edificações Comerciais, de Serviços e Públicas (RTQ-C) (INMETRO, 2012).

Há, entretanto, várias limitações e imprecisões encontradas na aplicação do RTQ-C (BAVARESCO et al., 2017; MELO et al., 2012; 2013; YAMAKAWA et al., 2011), o que motivou sua atualização, dando origem à Instrução Normativa INMETRO para Classe de Eficiência Energética de Edificações Comerciais, de Serviços e Públicas (INI-C). Esse novo regulamento prevê a atualização e a substituição do RTQ-C e passou por processo de Consulta Pública (INMETRO, 2018), não tendo sido ainda homologado.

Na INI-C são analisados os seguintes sistemas da edificação para fins de obtenção de ENCE: envoltória, condicionamento de ar, iluminação, equipamentos instalados, aquecimento de água e, apenas para fins informativos, percentual de economia devido uso racional de água (INMETRO, 2018). Nessa Instrução, cada sistema ou mesmo o edifício completo é classificado com base no percentual de economia de Energia Primária da edificação real em relação à edificação de referência, que é enquadrada na classe ' $D$ ' de eficiência energética (INMETRO, 2018). A Energia Primária é àquela disponível na natureza antes de sofrer qualquer transformação, contida nos combustíveis ainda brutos. Tal conceito foi introduzido pois, quando há diferentes fontes de energia (e.g., elétrica e térmica) sendo utilizadas para um uso final, é recomendável que a classificação de eficiência energética de um sistema leve em consideração todas as fontes de energia utilizadas, o que pode ser feito por fatores de conversão de energia (CB3E, 2017).

No novo regulamento há dois métodos para avaliação dos consumos: o método simplificado (Anexo B da INI-C) e o método de simulação (Anexo C da INI-C). Para aplicação do método simplificado da INI-C, os desenvolvedores criaram um endereço eletrônico para uso de um metamodelo que utiliza Redes Neurais Artificiais na obtenção da carga térmica anual de refrigeração, para as condições real e de referência da edificação em análise (CB3E, 2019). Tendo-se o valor da carga térmica, pode-se calcular o consumo de energia do sistema de condicionamento de ar, conforme equações expressas na INI-C. Também há outras equações 
para cálculo do consumo energético dos sistemas de iluminação, de aquecimento de água e dos equipamentos instalados.

No método de simulação da INI-C, houve melhoria em relação ao método de simulação do RTQ-C. No método antigo, o analista deveria modelar quatro edifícios de referência (níveis ' $A$ ' até ' $D$ ' de eficiência energética) e mais o edifício real. Na INI-C, há necessidade de modelagem apenas do edifício real e de um edifício de referência (Classe ' $\mathrm{D}$ '). Além disso, o Anexo A da INI-C traz tabelas com diversos parâmetros construtivos a serem adotados nesses dois modelos, enquanto no RTQ-C esses não eram expressos diretamente (deveriam ser calculados indiretamente por equações do método prescritivo).

Outra diretriz do Governo Federal, a qual também impacta diretamente os projetos elaborados por seus Órgãos Públicos, é a exigência do uso do Building Information Modelling (BIM), conforme Decreto ${ }^{\circ}$ 10.306, de abril (BRASIL, 2020). O Decreto dispôs que já a partir de janeiro de 2021, será exigido BIM na elaboração de modelos de arquitetura e de engenharia nas disciplinas de estrutura, hidráulica, AVAC (aquecimento, ventilação e ar condicionado) e elétrica, vinculando os ministérios da Defesa e da Infraestrutura.

Segundo Kamel et al. (2019), embora muitos projetistas usem ferramentas de simulação energética e ferramentas BIM, há um vazio de integração entre BIM e Building Energy Modelling (BEM) numa única ferramenta. A interoperabilidade é o caminho para essa comunicação, eliminando a necessidade de retrabalhos no preenchimento de dados e facilitando de forma automatizada o fluxo de trabalho entre diversos aplicativos usados no processo de projeto, conforme citam Andrade et al. (2009).

O Exército Brasileiro, como Órgão Público Federal, deve obter ENCE Geral Nível 'A' nos seus projetos e nas suas edificações construídas e ainda, elaborar todos os seus projetos utilizando a ferramenta BIM (TEIXEIRA, 2018). Entre 2019 e 2021 está sendo construído em Campo Grande, MS, um pavilhão militar já projetado em BIM atendendo ainda os requisitos para obtenção de ENCE nível ' $A$ ', quando avaliado pelo método prescritivo do RTQ-C (RACHID, 2018). Quando essa edificação foi projetada, não havia ainda sido publicada a Consulta Pública da INI-C. Além disso, na ocasião não foi integrado o projeto elaborado em BIM com a análise energética do empreendimento. Por isso, é recomendável uma nova avaliação conforme o novo regulamento, avaliando ainda a possibilidade do Exército utilizar modelos BIM para agilizar o processo de simulação.

O objetivo desse artigo é, portanto, aplicar o método de simulação da INI-C (INMETRO, 2018) para avaliação da eficiência energética de um Pavilhão do Exército Brasileiro projetado em BIM. Para permitir redução do tempo de análise de desempenho de projetos, indicou-se também uma metodologia a ser seguida no processo de interoperabilidade entre BIM e BEM, com estratégias gerais a serem aplicadas para avaliação de outros projetos de edificações militares. 


\section{METODOLOGIA}

\section{Caracterização do objeto de pesquisa}

O pavilhão militar avaliado é do tipo Companhia de Comando e Apoio (Pav CCAp), que é uma tipologia comum a praticamente todos os quartéis do Exército em todo o território nacional. A edificação apresenta formato ' $\mathrm{H}$ ' (Figura 1), sendo composto por uma edificação do lado leste e uma edificação do lado oeste, ligadas por uma circulação aberta nas laterais e com cobertura do mesmo material das edificações. Pode-se observar também os elementos de sombreamento utilizados no projeto (varandas voltadas para todas as fachadas, e existência de cobogós na fachada oeste de ambas as edificações que formam o pavilhão. Esses cobogós são elementos de sombreamento com disposição tal que bloqueie a insolação na fachada oeste).

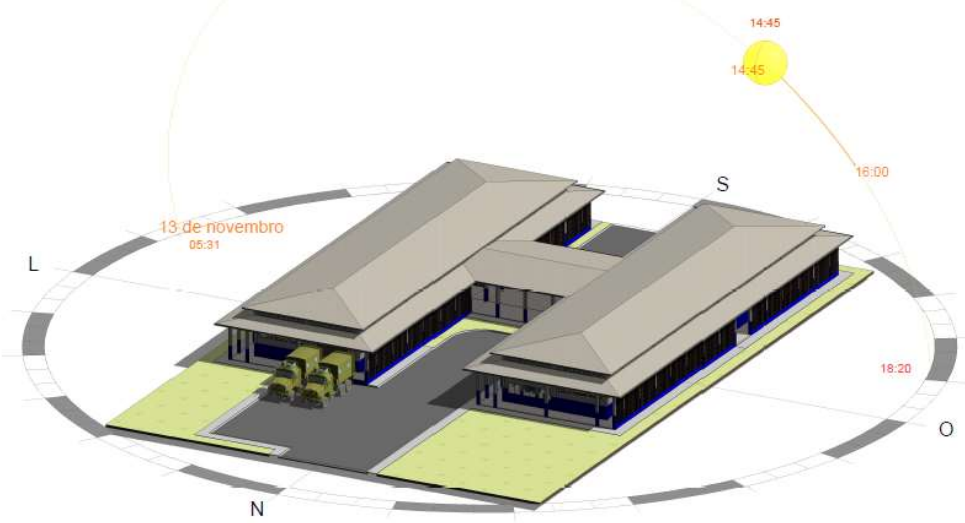

Figura 1: Vista em perspectiva do pavilhão do Exército Brasileiro avaliado pela INI-C. Fonte: CO/ $3^{\circ}$ GPT E (2017).

A área total construída da edificação do lado Leste é de 487,70 m², formada por depósitos, vestiários, banheiros e circulações. A maioria dos ambientes são de permanência transitória, não-condicionados artificialmente. A área total construída da edificação do lado Oeste é de 467,80 m², formada por escritórios, sala de aula e circulações. Desse lado, a maioria dos ambientes é de permanência prolongada, condicionados artificialmente. A tipologia predominante da edificação é de 'Escritórios' (conforme INI-C), com atividades complementares a essa.

A cobertura dos ambientes fechados do pavilhão é formada por laje pré-moldada de $12 \mathrm{~cm}$ (concreto com espessura de $4 \mathrm{~cm}$, EPS com espessura de $7 \mathrm{~cm}$ e argamassa com espessura de $1 \mathrm{~cm}$ ), uma laje técnica de 1,60 m de altura, e telhas termoacústicas TB-30, da camada mais interna para a mais externa. Os fechamentos externos são todos em alvenaria de bloco cerâmico $(9 \mathrm{~cm} \times 19 \mathrm{~cm} \times 19 \mathrm{~cm})$, com argamassa interna e externa de $2,5 \mathrm{~cm}$. A cor interna das paredes é branco gelo, e a externa é formada por cor azul escuro até $90 \mathrm{~cm}$ e branco acima disso. Por simplificação, foi considerado o valor de absortância solar $\alpha$ igual a 0,20 para as paredes externas (pintura na cor branca), conforme Tabela 1.3 do Manual para Aplicação do RTQ-C (INMETRO, 2016). As aberturas são compostas por vidro simples, não refletivo, com $6 \mathrm{~mm}$ de espessura e fator solar de 0,82 .

O sistema de iluminação é composto por luminárias com duas lâmpadas LED de 20 W e 2.600 lúmens cada. O sistema de condicionamento de ar dos ambientes condicionados artificialmente é composto por 
aparelhos do tipo Split Hi-Wall, Marca MIDEA ou equivalente técnico, de 9.000, 12.000 e 18.000 BTU/h, etiquetados pelo INMETRO com ENCE nível 'A' e COP médio de 3,24.

A edificação foi projetada para atender a Instrução Normativa no 02 (MPOG, 2014), quando avaliada pelo método prescritivo do RTQ-C (mas ainda sem submissão do projeto a um OIA - Organismo de Inspeção Acreditado).

\section{Clima e arquivos climáticos do local de pesquisa}

O clima de Campo Grande, MS, onde essa edificação militar está sendo construída, é tropical com inverno seco e verão chuvoso de acordo com a classificação de Köppen-Geiger.

Segundo a INI-C, podem ser utilizados diferentes arquivos climáticos para simulação, desde que sejam aprovados pelo laboratório de referência e que atendam algumas características, como fornecer dados horários de temperatura, umidade, radiação solar, direção e velocidade do vento (INMETRO, 2018).

Para a maioria dos meses, as médias são maiores de temperaturas de bulbo seco, de umidade relativa, e de radiação solar direta e difusa no arquivo climático SWERA (Solar and Wind Energy Resource Assessment) do que no INMET 2016 (Instituto Nacional de Meteorologia), conforme Figura 2 e Figura 3. Portanto, em todas as simulações, optou-se pela utilização do SWERA, que fornece resultados de consumo energético maiores na simulação (resultados, portanto, mais conservadores em uma análise energética).

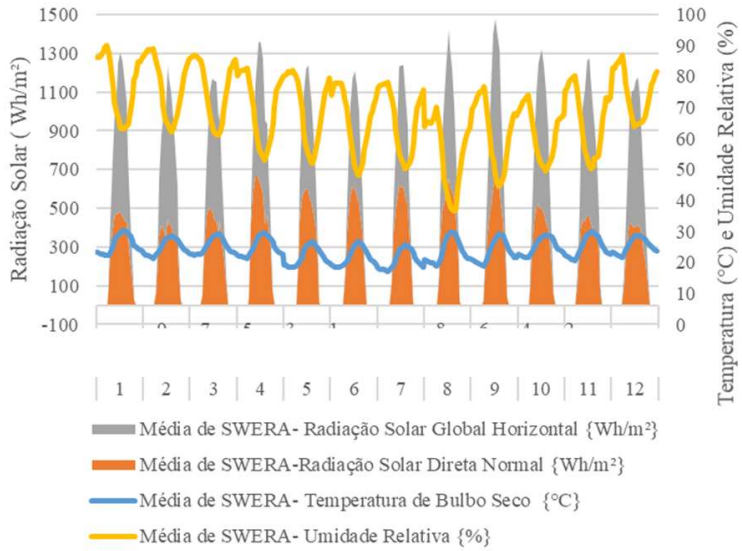

Figura 2: Temperatura de Bulbo Seco, Radiação Solar Direta e Global, e Umidade Relativa de Campo Grande, MS (SWERA).

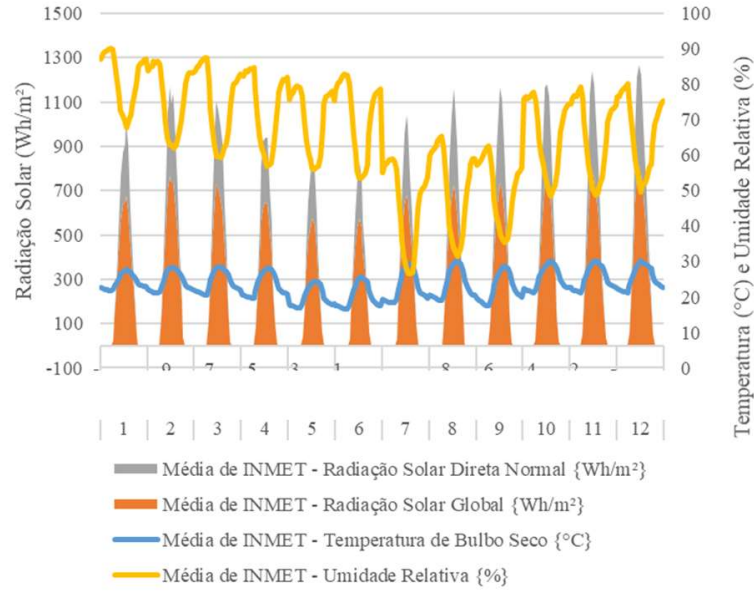

Figura 3: Temperatura de Bulbo Seco, Radiação Solar Direta e Global, e Umidade Relativa de Campo Grande, MS (INMET 2016).

\section{Método de Simulação da INI-C}

$\mathrm{Na}$ avaliação da edificação pelo método de simulação, por meio de softwares de simulação termoenergética de edificações, foi obtido o consumo final de energia elétrica e térmica dos sistemas individuais (condicionamento de ar, iluminação e equipamentos instalados). Comparou-se o consumo primário (simulado) do edifício na condição real com o consumo na condição de referência, obtendo-se a classificação energética da edificação.

Segundo a INI-C, podem ser utilizados diversos programas de simulação termoenergética, desde que atendam as algumas características previstas no Anexo $\mathrm{C}$ da INI-C. Assim, com o intuito de identificar um 
conjunto de ferramentas e metodologias mais adequadas à simulação termoenergética de edificações militares, foram analisadas três combinações: a) modelagem, caracterização e simulação termoenergética no software DesignBuilder versão 6.1.0.006, que utiliza como algoritmo o EnergyPlus versão 8.9.0.001 (

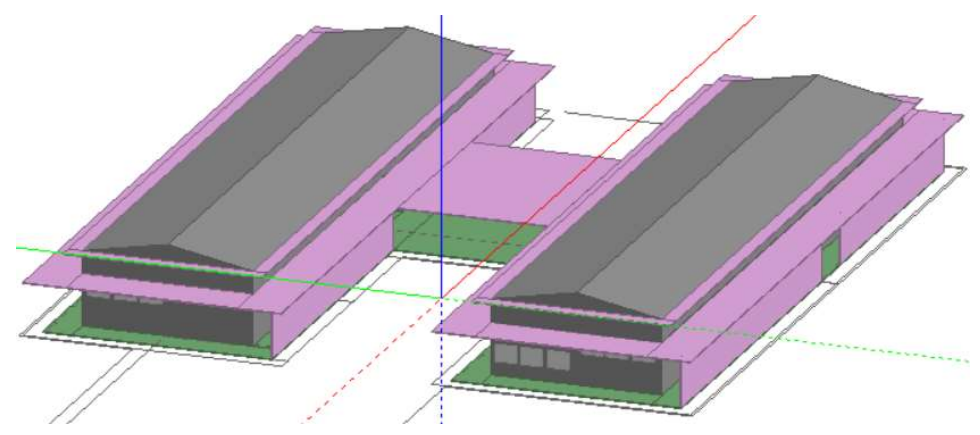

Figura 4); b) modelagem no software Revit 2019, caracterização e simulação termoenergética no software DesignBuilder versão 6.1.0.006 (Figura 5); c) modelagem nos softwares ScketchUp versão Pro 2017 e OpenStudio versão 2.8.0, caracterização e simulação termoenergética no software EnergyPlus versão 9.1.0 (Figura 6).

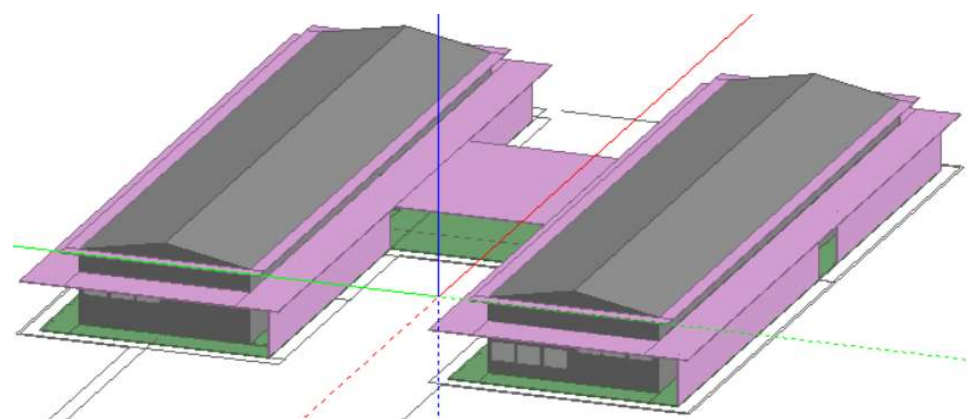

Figura 4: Modelagem da Edificação na Condição Real, no Software DesignBuilder.

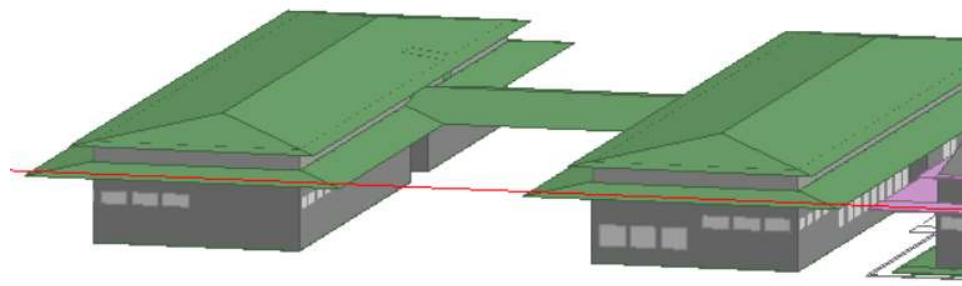

Figura 5: Modelagem da Edificação na Condição Real, no Software Revit posteriormente à importação para o DesignBuilder.

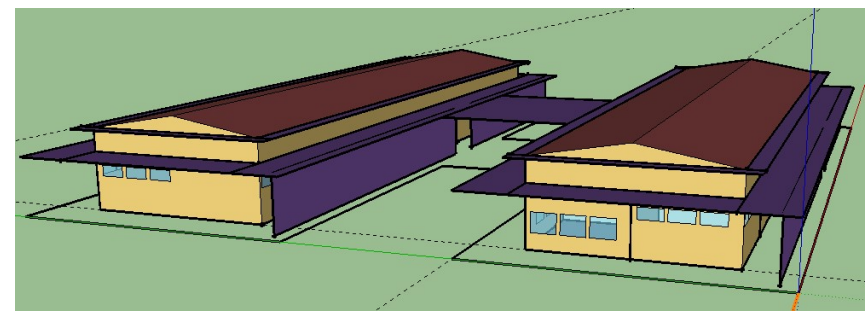

Figura 6: Modelagem da Edificação na Condição Real, no SkecthUp.

O EnergyPlus foi escolhido por ser utilizado em diversos trabalhos acadêmicos, por ser gratuito, além de ser recomendado pelo PROCEL Edifica e pela NBR 15.575 (ABNT, 2013). Porém, não apresenta interface amigável e a inserção de dados da edificação a ser simulada é relativamente complexa, gerando possíveis erros e dificultando sua utilização em larga escala pelo Exército. Como alternativa possível, é recomendada uma utilização em conjunto do EnergyPlus com o ScketchUp e com o OpenStudio, a qual foi realizada nesse 
trabalho (GOUVEIA et al., 2014; SILVA, 2010).

Pimentel et al. (2020) analisaram outras possibilidades de softwares de simulação, principalmente os que apresentavam possibilidade de interoperabilidade com o Revit, uma vez que o Exército utiliza esse último como ferramenta de projetos em BIM. O DesignBuilder se destacou por apresentar interoperabilidade facilitada com o Revit, por utilizar o mesmo algoritmo do EnergyPlus, e por permitir a modelagem na edificação inteiramente no próprio programa caso necessário, sendo, pois, adotado nesse trabalho.

Para a modelagem da edificação analisada, nas condições real e de referência, as características como transmitância térmica e absortância solar das paredes e coberturas, propriedades dos vidros, Percentual de Abertura na Fachada, Ângulos Horizontais e Verticais de Sombreamento, Coeficiente de Performance do sistema de condicionamento de ar, e Densidade de Potência de lluminação, dentre outras, foram definidas conforme tabelas do Anexo A da INI-C para cada condição. A Erro! Fonte de referência não encontrada., por exemplo, expressa condições e valores que devem ser adotados, para a tipologia de escritórios.

Tabela 1: Valores para avaliação energética de edificações de escritórios pela INI-C.

\begin{tabular}{|c|c|c|}
\hline Classe & Variáveis & Referência \\
\hline Geometria & $\begin{array}{l}\text { Forma } \\
\text { Orientação Solar }\left({ }^{\circ}\right) \\
\text { Pé-direito }(\mathrm{m}) \\
\text { AHS - Ângulo Horizontal de Sombreamento }\left({ }^{\circ}\right) \\
\text { AVS - Ângulo Vertical de Sombreamento }\left(^{\circ}\right) \\
\text { AOV - Ângulo de Obstrução Vertical }\left(^{\circ}\right)\end{array}$ & $\begin{array}{l}\text { Condição Real } \\
\text { Condição Real } \\
\text { Condição Real } \\
0 \\
0 \\
\text { Condição Real (opcional) }\end{array}$ \\
\hline Aberturas & $\begin{array}{l}\text { PAF - Percentual de Abertura da Fachada (\%) } \\
\text { PAZ - Percentual de Abertura Zenital (\%) }\end{array}$ & $\begin{array}{l}50,00 \\
0,00\end{array}$ \\
\hline Componentes Construtivos & $\begin{array}{l}\mathrm{U}_{\mathrm{par}} \text { transmitância térmica }\left(\mathrm{W} / \mathrm{m}^{2} \mathrm{~K}\right) \text { paredes externas } \\
\alpha_{\mathrm{par}}-\text { absortância térmica (adimensional) paredes externas } \\
\mathrm{CT}_{\mathrm{par}}-\text { Capacidade Térmica }\left(\mathrm{kJ} / \mathrm{m}^{2} \mathrm{~K}\right) \text { paredes externas } \\
\mathrm{U}_{\mathrm{cob}} \text { - transmitância térmica }\left(\mathrm{W} / \mathrm{m}^{2} \mathrm{~K}\right) \text { cobertura } \\
\alpha_{\mathrm{cob}}-\text { absortância térmica (adimensional) cobertura } \\
\mathrm{CT}_{\mathrm{cob}}-\text { Capacidade Térmica }\left(\mathrm{kJ} / \mathrm{m}^{2} \mathrm{~K}\right) \text { cobertura } \\
\mathrm{FS} \text { - Fator Solar (adimensional) vidros } \\
\mathrm{U}_{\text {vid }} \text { - transmitância térmica }\left(\mathrm{W} / \mathrm{m}^{2} \mathrm{~K}\right) \text { vidros }\end{array}$ & $\begin{array}{l}2,39 \\
0,50 \\
150,00 \\
2,06 \\
0,80 \\
233,00 \\
0,82 \\
5,70\end{array}$ \\
\hline Iluminação e ganhos & $\begin{array}{l}\text { DPI - Densidade de Potência de lluminação }\left(\mathrm{W} / \mathrm{m}^{2}\right) \\
\text { Ocupação ( } \mathrm{m}^{2} / \text { pessoa) } \\
\text { DPE - Densidade de Potência de Equipamentos }\left(\mathrm{W} / \mathrm{m}^{2}\right) \\
\text { Horas de ocupação (horas) } \\
\left.\text { Dias de ocupação ( } \mathrm{N}_{\text {ano }}\right) \\
\text { Condição do piso } \\
\text { Condição da cobertura } \\
\text { Isolamento do piso }\end{array}$ & $\begin{array}{l}14,10 \\
10,00 \\
9,70 \\
10,00 \\
260,00 \\
\text { Condição real } \\
\text { Condição real } \\
\text { Sem isolamento }\end{array}$ \\
\hline Condicionamento de ar & $\begin{array}{l}\text { COP - Coeficiente de Performance }(\mathrm{W} / \mathrm{W}) \\
\text { Temperatura setpoint }\left({ }^{\circ} \mathrm{C}\right) \\
\text { Aquecimento de água }\end{array}$ & $\begin{array}{l}2,60 \\
24,00 \\
\text { Não significativo }\end{array}$ \\
\hline
\end{tabular}

Fonte: INMETRO (2018).

Considerou-se a ocupação da edificação nos horários de 08 h00min às 18 h00min, de segunda a sexta, nos quais funcionam todos os sistemas (iluminação, equipamentos e condicionamento de ar). Como simplificação, considerou-se $100 \%$ de carga de cada ganho térmico interno em todas utilizadas, de acordo com Versage (2015). Os fins de semana foram considerados sem ocupação. Este tipo de utilização está de acordo com o padronizado no Anexo A da INI-C para edificações de escritório.

De acordo com Versage (2015), considerou-se o total de ganho de calor por pessoa de $120 \mathrm{~W}$, 
condizente com uma atividade média de escritório desempenhada pelos ocupantes. Fixou-se a fração radiante dos ganhos de calor sensível pelas pessoas em $30 \%$, sendo o restante de $70 \%$ assumido como carga térmica por convecção. Fixou-se a fração radiante das cargas internas do sistema de iluminação e dos equipamentos em $72 \%$ e a fração convectiva em $10 \%$, sendo o restante de $18 \%$ a fração visível das lâmpadas ou a fração de trabalho dos equipamentos. Na modelagem, para minimizar divergências entre os resultados obtidos com os softwares utilizados, em decorrência de possível diferença na área útil dos ambientes, optouse por utilizar valores de ocupação, de equipamentos e de iluminação em números absolutos, não em densidade.

Para modelagem do sistema de condicionamento de ar, foram utilizados objetos Packaged Terminal Air Conditioner (PTAC). A modelagem por esses objetos exige alguns parâmetros de entrada específicos, os quais os principais foram expressos na Tabela 2. No caso do PTAC, pode-se obter como dado de saída o consumo energético anual do sistema (com COP real variável em função da operação do sistema) e a carga térmica anual.

Tabela 2: Dados de entrada do objeto PTAC para modelagem de sistema de condicionamento de ar.

\begin{tabular}{|c|c|}
\hline Parâmetro & PTAC \\
\hline \multirow{8}{*}{ Características gerais da modelagem } & Modelagem de um sistema para cada Zona Térmica \\
\hline & Setpoint $\left(24^{\circ} \mathrm{C}\right)$ \\
\hline & Disponibilidade do sistema de aquecimento (sempre desligado) \\
\hline & Disponibilidade do sistema de resfriamento ( 8 h00-18h00 de segunda a sexta) \\
\hline & Temperatura mínima de insuflamento de ar para resfriamento $\left(13^{\circ} \mathrm{C}\right)$ \\
\hline & Método de cálculo de vazão externa de ar (vazão por área) \\
\hline & Vazão externa de ar por área $\left(0,0004 \mathrm{~m}^{3} / \mathrm{s}-\mathrm{m}^{2}\right)$ \\
\hline & Controle de insuflamento de ar constante \\
\hline Fator de dimensionamento de resfriamento & 1,15 \\
\hline Auto dimensionamento & Capacidade (W) e fluxo de $\operatorname{ar}\left(\mathrm{m}^{3} / \mathrm{s}\right)$ \\
\hline $\begin{array}{l}\text { Posicionamento dos ventiladores para } \\
\text { insuflamento }\end{array}$ & Draw through \\
\hline $\begin{array}{l}\text { Eficiência total dos ventiladores para insuflamento } \\
\text { de ar }\end{array}$ & $65 \%$ \\
\hline Pressão de ar dos ventiladores para insuflamento & $250 \mathrm{~Pa}$ \\
\hline $\begin{array}{l}\text { Eficiência do motor dos ventiladores para } \\
\text { insuflamento }\end{array}$ & $90 \%$ \\
\hline COP médio do sistema bruto de refrigeração & 3,24 \\
\hline Tipo do sistema bruto de refrigeração & Elétrico \\
\hline
\end{tabular}

\section{Obtenção do Consumo Anual Total e da ENCE Geral de Projeto}

Após avaliação desses sistemas individualmente, obteve-se o consumo total anual da edificação pela Equação Eq. 1.

$$
C T E_{E}=C I L+C C A_{E}+C E Q
$$

Onde:

$C T E_{E}$ é o consumo anual total de energia elétrica da edificação ( $\mathrm{kWh} / \mathrm{ano}$ ); $C I L$ é o consumo anual total de energia elétrica do sistema de iluminação (kWh/ano); $C C A_{E}$ é o consumo anual total de energia elétrica do sistema de condicionamento de ar (kWh/ano); $C E Q$ é o consumo anual total de energia elétrica dos equipamentos instalados ( $\mathrm{kWh} / \mathrm{ano}$ ).

Após o cálculo do consumo energético anual total, obteve-se o consumo total anual de energia primária da edificação, conforme a Equação Eq. 2.

$$
C E P_{\text {REAL OU REF }}=f_{C E} \times C T E_{E}+f_{C T} \times C T E_{T} \quad \text { Eq. } 2
$$


$C E P_{R E A L}$ OU REF é o consumo total de energia primária da edificação, que pode ser em sua condição real (CEPREAL $\mathrm{kWh} / \mathrm{ano}$ ) ou de referência (CEP $\left.\mathrm{REF}_{\mathrm{R}}-\mathrm{kWh} / \mathrm{ano}\right)$; $f_{C E}$ é o fator de conversão de energia elétrica para energia primária, igual a 1,6;

$C T E_{E}$ é o consumo total anual de energia elétrica da edificação avaliada (kWh/ano), obtido pela Equação Eq. 1; $f_{C T}$ é o fator de conversão de energia térmica para energia primária, igual a 1,1, tanto para gás natural, como para gás liquefeito de petróleo;

$C T E_{T}$ é o consumo total anual de energia térmica da edificação avaliada (kWh/ano), quando existente. Para essa edificação, não há fontes térmicas.

Finalmente, calculou-se o percentual de economia de energia da edificação real em relação à edificação de referência, o que se fez pela Equação Eq. 3:

$$
P_{R C E P_{R E A L-D}}=1-\frac{C E P}{C E P_{R E F}} * 100
$$

Onde:

$P R C E P_{R E A L-}$ é o percentual de redução do consumo de energia primária entre a condição real e de referência; $C E P$ é o consumo anual de energia primária da edificação na condição real ( $\mathrm{kWh} / \mathrm{ano})$; $C E P_{R E F}$ é o consumo anual de energia primária da edificação na condição de referência (kWh/ano). Para essa edificação, o lado Leste apresenta Fator de Forma de 0,74 e o lado Oeste, de 0,77. Uma vez que o Fator de Forma dessa edificação de escritório é maior que 0,50, para a cidade de Campo Grande, MS Grupo Climático 10 pela classificação de Roriz (2014) - pela Tabela 5 da INI-C (INMETRO, 2018), obtêm-se que o coeficiente de redução mínimo do consumo de energia primária da classe ' $D$ ' para a classe 'A' é de $38 \%$. Se a economia é maior que essa, classifica-se a edificação com ENCE Geral classe 'A'. Caso contrário, deve-se preencher uma tabela com intervalos de eficiência para obtenção de classes 'B', 'C ', 'D' ou 'E', o que não foi necessário.

\section{Observações sobre a exportação do modelo BIM (Revit) para o DesignBuilder}

O Exército utiliza o software Autodesk Revit para elaboração dos seus projetos. Apesar do modelo BIM conter informações sobre a volumetria e sobre alguns componentes construtivos de uma edificação, não é possível uma transição direta do Revit para o DesignBuilder. Portanto, seguiu-se a sequência a seguir (Figura 7).

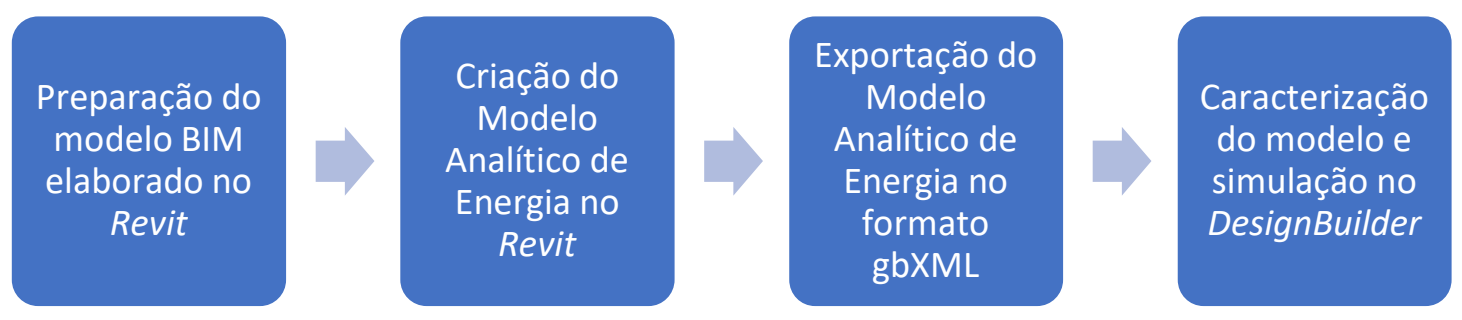

Figura 7: Etapas de interoperabilidade entre o Revit e o EnergyPlus.

Um modelo de simulação deve ter complexidade apenas suficiente para atender o objetivo pelo qual é proposto, que é representar uma realidade em 'experimentos virtuais', revelando o comportamento resultante de cada diretriz adotada e quantificando os resultados (HENSEN et al., 2011). Assim, de todas as informações contidas no modelo Revit, foram mantidas apenas as necessárias para simulação termoenergética: divisão em zonas térmicas (chamadas de ambientes no Revit), janelas, telhados, envoltória (paredes, pisos e lajes). Elementos como pilares, vigas, lajes, mobiliários e peças hidráulicas foram excluídos, por terem gerados erros de interoperabilidade, conforme ilustrado na Erro! Fonte de referência não 
encontrada.. Essa exclusão deve ser feita antes da criação do Modelo Analítico de Energia, ainda na preparação do modelo BIM.

Conforme INI-C, não devem ser avaliados os ambientes de permanência transitória não condicionados artificialmente (que na edificação em análise são corredores, depósitos, sanitários e outras áreas não ocupadas). Para essas áreas, devem ser computados apenas o consumo de eletricidade do sistema de iluminação, desconsiderando consumo de equipamentos e de condicionamento de ar. Portanto, tais áreas podem ser agrupadas no modelo Revit antes da criação do Modelo Analítico de Energia. Assim, foram excluídas as paredes que separavam esses ambientes, agrupando-os, conforme Erro! Fonte de referência não encontrada. e Figura 10.

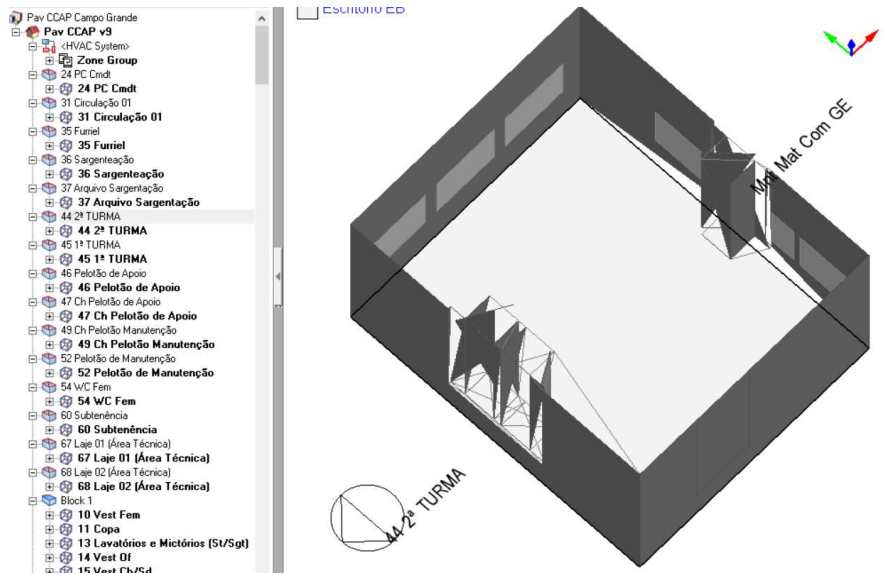

Figura 8: Importação da Edificação sem exclusão de elementos estruturais, mobiliários e peças hidráulicas, no DesignBuilder.

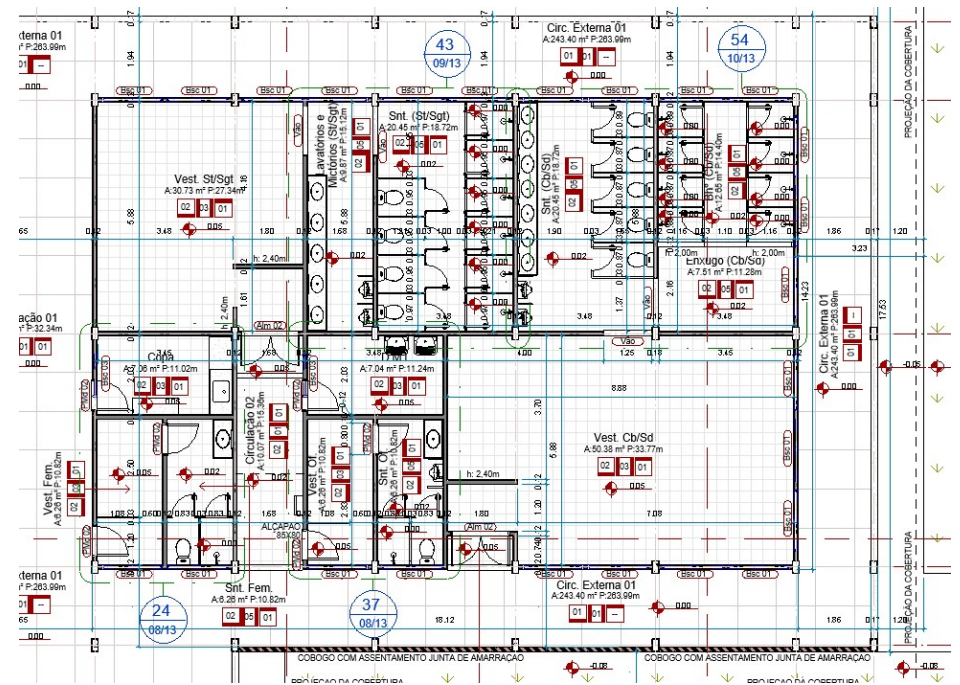

Figura 9: Planta baixa original do Pavilhão militar, edificação do lado Leste, no Revit. Fonte: CO/ $3^{\circ}$ GPT E (2017). 


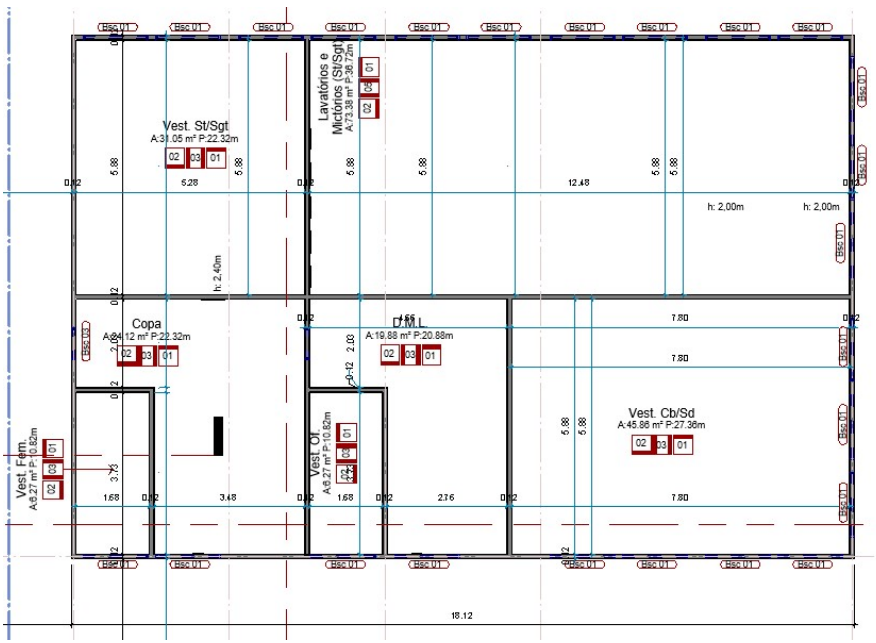

Figura 10: Planta baixa do Pavilhão militar, edificação do lado Leste, no Revit, após exclusão de elementos e agrupamento de ambientes de permanência transitória.

Após todas essas simplificações no projeto elaborado em Revit, no próprio programa optou-se por exportar o modelo no formato gbXML, o qual é compatível com o DesignBuilder. Para isso, foi criado no Revit um Modelo Analítico de Energia, que é uma representação das zonas térmicas formadas pelos cômodos fechados do projeto (no Revit chamam-se ambientes). Esse modelo, e não o projeto em si, é de fato exportado. Portanto, antes de se proceder com a exportação, foram corrigidas as zonas térmicas que apresentaram erros. Esses erros ocorreram quando não foram totalmente excluídos elementos estruturais (como vigas), que impediram o total fechamento de algumas zonas térmicas (havia espaços entre as paredes e os tetos). Também ocorreram irregularidades na envoltória (reentrâncias) que puderam ser observadas e corrigidas antes da exportação para o DesignBuilder.

Foi muito importante a utilização da própria ferramenta de visualização de zonas do Modelo Analítico de Energia (Figura 11), que permite a análise de cada superfície do modelo para detecção de possíveis erros antes da efetiva exportação. Na Figura 11, destaca-se, na aba detalhes, o símbolo de advertência em amarelo com um sinal de exclamação, o qual indica que uma zona térmica não está totalmente fechada, exigido correções no projeto para criação posterior de um novo Modelo Analítico de Energia.

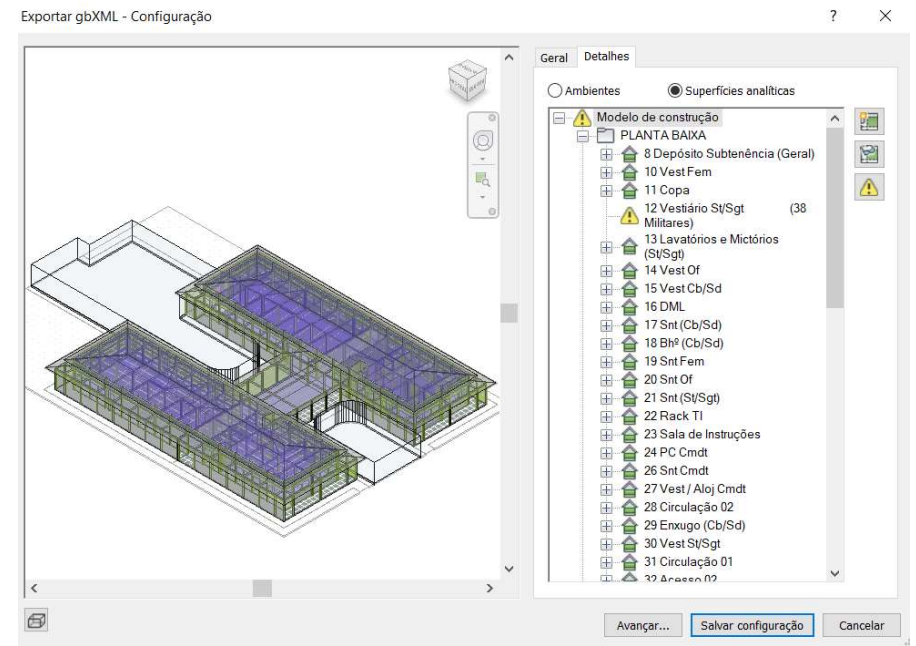

Figura 11: Ferramenta do Revit que permite conferência das superfícies e zonas térmicas do Modelo Analítico de Energia antes da exportação. 
Após total conferência do Modelo Analítico de Energia, exportou-se o modelo na extensão gbXML para leitura no DesignBuilder (Figura 5). Na última fase, caracterizou-se o modelo no DesignBuilder seguindo as diretrizes para simulação estabelecidas pela INI-C, já apresentadas nesse trabalho, e processou-se a simulação propriamente dita.

\section{RESULTADOS E DISCUSSÃO}

\section{Consumo energético anual para condição Real}

A Erro! Fonte de referência não encontrada. expressa os consumos energéticos obtidos, para cada combinação de softwares proposta. O consumo total se deu por soma (Equação 1) dos consumos individuais do sistema de condicionamento de ar, do sistema de iluminação e dos equipamentos instalados, todos avaliados em cada um dos cenários propostos.

Pela Erro! Fonte de referência não encontrada., observa-se que o consumo total obtido pelo EnergyPlus (com modelagem em ScketchUp) e pelo DesignBuilder (com modelagem em Revit) obtiveram desvio de 0,03\%, com excelente correlação. Ambos apresentaram diferença de aproximadamente 1,00\% em relação ao consumo total obtido pelo DesignBuilder (com modelagem no próprio software).

No caso dos equipamentos instalados e do sistema de iluminação, os resultados obtidos por todas as combinações entre ferramentas foram iguais. Isso ocorreu porque foram calculados, para cada zona térmica, valores absolutos de potência instalada com base na área de piso real de projeto. Tal decisão foi importante, uma vez que o Revit utiliza a área de piso conforme projeto, o que não ocorre de maneira exata na modelagem realizada no ScketchUp (para simulação no EnergyPlus) e na modelagem no DesignBuilder.

Tabela 3: Consumo energético anual (kWh/ano) na Condição Real (conforme projeto).

\begin{tabular}{|c|c|c|c|c|c|c|}
\hline Sistema Avaliado (kWh/ano) & $\begin{array}{l}\text { EnergyPlus } \\
(E+)\end{array}$ & $\begin{array}{l}\text { DesignBuilder } \\
\text { (DB) }\end{array}$ & $\begin{array}{l}\text { Revit } \\
\text { DesignBuilder } \\
\text { (Revit) }\end{array}$ & ${ }^{+} \% \mathrm{DB}-\mathrm{E}+$ & \% Revit - E+ & \% Revit - DB \\
\hline $\begin{array}{llll}\text { Envoltória ( Carga Térmica de } \\
\text { Resfriamento) }\end{array}$ & $33.069,56$ & $33.887,31$ & $32.595,17$ & $2,47 \%$ & $-1,43 \%$ & $-3,81 \%$ \\
\hline Sistema de Iluminação & $9.291,60$ & $9.291,60$ & $9.291,60$ & $0,00 \%$ & $0,00 \%$ & $0,00 \%$ \\
\hline Equipamentos Instalados & $12.967,62$ & $12.967,62$ & $12.967,36$ & $0,00 \%$ & $0,00 \%$ & $0,00 \%$ \\
\hline Sistema de Condicionamento de $\mathrm{Ar}$ & $9.881,51$ & $10.198,53$ & $9.872,86$ & $3,21 \%$ & $-0,09 \%$ & $-3,19 \%$ \\
\hline Consumo Total & $32.140,73$ & $32.457,75$ & $32.131,83$ & $0,99 \%$ & $-0,03 \%$ & $-1,00 \%$ \\
\hline
\end{tabular}

No ScketchUp, há uma maior exatidão do modelo em relação às zonas térmicas de projeto, uma vez que a modelagem foi elaborada zona por zona por cima da planta da edificação importada no formato Drawing Exchange Format (DXF). A modelagem foi realizada zona por zona pois foi utilizado o software OpenStudio para compatibilizar as superfícies de cada zona entre si (por exemplo: paredes, lajes, aberturas) antes da importação para o EnergyPlus, no qual foi processada a simulação propriamente dita.

Essa característica específica da modelagem no ScketchUp justifica a aproximação com os resultados obtidos com a simulação do projeto importado do Revit no DesignBuilder. Isso porque as zonas térmicas são construídas com volumes praticamente iguais nas duas combinações, resultando em consumos energéticos muito próximos (0,03\% de diferença).

A diferença $(1,00 \%)$ dessas duas combinações em relação ao modelo simulado no DesignBuilder, 
porém modelado no próprio software, se explica pelo fato da modelagem ser elaborada de uma forma distinta no último caso. Assim como no ScketchUp, importa-se no DesignBuilder a planta da edificação no formato DXF. Porém, deve-se modelar primeiramente a envoltória total externa da edificação, somente posteriormente definindo as paredes internas, por cima da planta em DXF. Assim, na definição das paredes internas para divisão das zonas térmicas, há construção de cada parede no seu eixo médio, provocando mudanças de volume das zonas térmicas adjacentes em relação ao volume real da edificação projetada. Essas diferenças volumétricas geram diferenças no cálculo de carga térmica de resfriamento, refletindo, pois, no consumo anual de energia simulado.

Dessas diferenças de modelagem decorre a importância de se calcular previamente valores absolutos para potência de equipamentos e iluminação, como se fez, e não definir nos softwares de simulação apenas valores de densidade de potência (conforme diretrizes da Tabela 1), que gerariam valores distintos de consumo energético simulado pelo fato das modelagens apresentarem áreas de piso diferentes.

\section{Consumo energético anual para condição de Referência}

A Tabela 4 expressa os consumos energéticos obtidos, para combinação de softwares proposta, na condição de Referência. A modelagem da edificação nessa condição obedeceu a todas diretrizes expressas na Tabela 1. O consumo total se deu por soma (Equação 1) dos consumos individuais do sistema de condicionamento de ar, do sistema de iluminação e dos equipamentos instalados, todos avaliados em cada um dos cenários propostos.

Tabela 4: Consumo energético anual (kWh/ano) na Condição de Referência.

\begin{tabular}{lllllll}
\hline Sistema Avaliado (kWh/ano) & $\begin{array}{l}\text { EnergyPlus } \\
\text { (E+) }\end{array}$ & $\begin{array}{l}\text { DesignBuilder } \\
\text { (DB) }\end{array}$ & $\begin{array}{l}\text { Revit } \\
\text { DesignBuilder } \\
\text { (Revit) }\end{array}$ & \% DB - E+ \% Revit - E+ \% Revit - DB \\
\hline $\begin{array}{l}\text { Envoltória ( Carga Térmica de } \\
\text { Resfriamento) }\end{array}$ & $80.876,36$ & $80.273,23$ & $80.265,37$ & $-0,75 \%$ & $-0,76 \%$ & $-0,01 \%$ \\
\hline Sistema de lluminação & $36.534,56$ & $36.534,56$ & $36.534,56$ & $0,00 \%$ & $0,00 \%$ & $0,00 \%$ \\
\hline Equipamentos Instalados & $12.967,62$ & $12.967,62$ & $12.967,36$ & $0,00 \%$ & $0,00 \%$ & $0,00 \%$ \\
\hline Sistema de Condicionamento de Ar & $30.611,28$ & $30.619,97$ & $30.618,60$ & $0,03 \%$ & $0,02 \%$ & $0,00 \%$ \\
\hline Consumo Total & $80.113,46$ & $80.122,15$ & $80.120,53$ & $0,01 \%$ & $0,01 \%$ & $0,00 \%$ \\
\hline
\end{tabular}

Pela Tabela 4, observa-se que o consumo total obtido pelo EnergyPlus (com modelagem em ScketchUp), pelo DesignBuilder (com modelagem em Revit) e pelo DesignBuilder (com modelagem no próprio software) apresentaram desvios máximos de 0,01\% entre si, uma excelente correlação.

No caso dos equipamentos instalados e do sistema de iluminação, os resultados obtidos por todas as combinações entre ferramentas foram iguais. Isso ocorreu porque foram calculados, para cada zona térmica, valores absolutos de potência instalada com base na área de piso real de projeto, tal como foi feito na modelagem da edificação na condição real, pelo mesmo motivo já apresentado.

A edificação de referência, conforme diretrizes da INI-C (Tabela 1), não apresenta elementos de sombreamento e o Percentual de Abertura de Fachada é fixado em $50 \%$. Assim, as três modelagens tornamse praticamente iguais na condição de referência, o que não ocorre na modelagem da edificação real, que segue o projeto, com suas janelas representando percentuais de abertura distintos em relação a cada 
fachada e com seus elementos de sombreamento representando diferentes ângulos de sombreamento em relação a cada janela.

Essa semelhança maior na modelagem da edificação de referência, por ter menos complexidade arquitetônica e por ter elementos mais homogêneos, explica o fato de as diferenças entre as três combinações de softwares ter se tornado quase nula $(0,01 \%)$.

\section{ENCE Geral de Projeto}

Em relação ao edifício real, por todas combinações analisadas, o edifício de referência (equivalente à classe ' $D$ ' de eficiência energética) apresentou maior consumo total anual de energia. Os percentuais de economia do edifício real em relação ao de referência, utilizados para obtenção da ENCE Geral de projeto, estão representados na Tabela 5 e foram calculados pela Equação 3.

Como há apenas fontes de energia elétrica na edificação avaliada, o percentual de economia de energia primária coincide com o percentual de economia de energia elétrica. Assim, como todas as economias ficaram acima de 38\% (valor do coeficiente de redução do consumo de energia primária da classe 'D' para a classe 'A', para o Grupo Climático 10), por todas combinações foi obtida ENCE Geral de Projeto classe ' $A$ '.

Tabela 5: Percentual de Economia (consumo energético anual do Edifício Real em relação ao do Edifício de Referência) para obtenção de ENCE Geral de Projeto.

\begin{tabular}{|c|c|c|c|c|}
\hline Sistema Avaliado (kWh/ano) & EnergyPlus (E+) & DesignBuilder (DB) & $\begin{array}{l}\text { Revit }+ \\
\text { (Revit) }\end{array}$ & DesignBuilder \\
\hline $\begin{array}{l}\text { Percentual de Economia Edifício Real em relação ao de } \\
\text { Referência }\end{array}$ & $59,88 \%$ & $59,49 \%$ & $59,90 \%$ & \\
\hline ENCE Geral de Projeto & Classe 'A' & Classe 'A' & Classe ' $A$ ' & \\
\hline
\end{tabular}

Portanto, apesar das diferenças entre os consumos energéticos obtidos, independentemente da combinação de softwares que se utilize, não há prejuízo à ENCE. Pela Tabela 3 e pela Tabela 4, todas as três opções apresentaram praticamente a mesma precisão (desvios máximos de 1\% quanto aos consumos). Assim, a escolha do software a ser utilizado para aplicação em larga escala pelo Exército pode ser feita considerando outros fatores (uma vez que há semelhança de precisão), como custo dos softwares, tempo e complexidade do processo de modelagem e simulação.

\section{Complexidade do processo de modelagem}

Na simulação pelo EnergyPlus, primeiramente foi necessário a modelagem de toda a edificação no ScketchUp em conjunto com o OpenStudio. Para cada superfície desenhada no ScketchUp (parede, porta, janela, laje, cobertura), deve-se configurar o tipo de superfície no OpenStudio, compatibilizando paredes de zonas térmicas adjacentes. Assim, para cada cômodo desenhado, deve-se, parede por parede, por exemplo, atribuir manualmente a vizinhança com paredes de cômodos vizinhos modelados anteriormente.

A modelagem dessa edificação totalizou 27 zonas térmicas, 221 superfícies, 22 janelas, 21 elementos de sombreamento e para cada um desses elementos teve-se que fazer as devidas configurações no ScketchUp e no OpenStudio, o que dificultou o processo. Houve também muitos travamentos nos softwares 
devido à complexidade do modelo, causando necessidade de retrabalho algumas vezes. Ressalta-se que, ainda, todo esse processo teve que ser repetido para edificação de referência.

Uma vez finalizada a modelagem, exportou-se o modelo para o EnergyPlus. Nesse software, foram definidos todos os elementos construtivos (tipos de parede, janelas, telhados), as características de ocupação (dias e horas de ocupação, calor emitido pelos usuários), e as características dos sistemas instalados (ar condicionado e iluminação). O processo só pode ser feito manualmente, para cada elemento, e para cada zona térmica.

Portanto, no quesito precisão, nota-se o potencial do uso conjunto do EnergyPlus com ScketchUp e OpenStudio, combinação que ofereceu resultados com no máximo 1,00\% de diferença em relação a utilização do software pago DesignBuilder, mas com o EnergyPlus e OpenStudio sendo ferramentas gratuitas. Porém, para uma aplicação coorporativa e em larga escala, como é a utilização pelo Exército, torna-se inviável essa combinação, pelo tempo aplicado de 70 horas, em média, e pela complexidade do processo.

Na modelagem e simulação realizada no DesignBuilder, por outro lado, há algumas facilidades. O próprio software dispõe de uma ferramenta de desenho que permite a modelagem de toda a edificação. Durante a modelagem, basta que se desenhe a edificação e depois que se faça a divisão interna das zonas térmicas (por meio de paredes). O próprio software atribui automaticamente todas condições de adjacências entre as superfícies.

Uma vez finalizada a modelagem, inicia-se a fase de caracterização do modelo no DesignBuilder, que precede a simulação. Também há diversas vantagens em relação ao uso dos softwares da combinação anterior, como tabelas de fácil visualização que facilitam a modelagem dos elementos construtivos, das características de ocupação e dos sistemas instalados. O DesignBuilder também permite que as mudanças sejam atribuídas à um elemento específico, a uma zona inteira, ou mesmo para toda a edificação. Tal possibilidade evitou a necessidade de, para cada uma das 221 superfícies do caso estudado, se ter que fazer a definição de características. Características que sejam, por exemplo, comuns a toda a edificação, podendo ser definidas uma única vez e atribuídas a todo o modelo. O tempo médio aplicado nessa modelagem foi de 52 horas.

Após a simulação, a saída de dados no DesignBuilder pode ser agrupada em gráficos e tabelas de fácil visualização e de alternância entre si facilitada, ao contrário do EnergyPlus, para o qual a saída é estritamente a que foi escolhida e, caso se queira outro dado, é necessário executar novamente a simulação.

Por fim, a última combinação analisada foi a utilização de um projeto elaborado no Revit com caracterização e simulação no DesignBuilder. Essa opção foi a mais vantajosa de todas, uma vez que o Exército já elabora seus projetos utilizando o Revit, e esses projetos podem ser exportados para o DesignBuilder, evitando o retrabalho com o processo de modelagem que antecede a simulação. Porém, cabe salientar que, conforme descrito no Método, o projeto inicial deve passar por um processo prévio de simplificação (exclusão de elementos estruturais e de algumas zonas térmicas), criando-se o Modelo Analítico de Energia, o qual é de fato exportado para o DesignBuilder. Uma vez no DesignBuilder, o processo de caracterização é o mesmo já descrito anteriormente. 
Essa terceira opção foi menos trabalhosa que as demais e eliminou a necessidade da modelagem digital para simulação de um projeto que já existe em BIM, e que, por meio de interoperabilidade, pode ser reaproveitado. Assim, é perfeitamente possível que o Exército Brasileiro, que atende ao Decreto $\mathrm{n}^{\circ} 10.306$ (BRASIL, 2020), o qual exige utilização de BIM, e à IN nº 02 (MPOG, 2014), a qual obriga obtenção de ENCE classe ' $A$ ' para projetos, possa agilizar o processo de análise energética de seus empreendimentos, com a interoperabilidade entre o BIM e o BEM. O tempo médio empregado nessa modelagem foi de 39 horas.

\section{CONCLUSÕES}

O Exército Brasileiro deve obter ENCE Geral de Projeto nível 'A' e deve utilizar a metodologia BIM para todas as suas edificações que serão (ou que estão sendo) construídas. O projeto do pavilhão tomado para estudo nesse artigo foi elaborado utilizando BIM no software Autodesk Revit e obteve ENCE Geral de Projeto nível 'A', quando avaliado pelo método prescritivo do RTQ-C. Porém, foi divulgado em 2018 o texto da INI-C, que substituirá o RTQ-C, com novos métodos para avaliação de eficiência energética de edificações públicas.

Assim, buscando aproveitar a possibilidade de integração entre o BIM e ferramentas de simulação termoenergética de edificações, foi realizada uma análise energética desse pavilhão militar utilizando três combinações entre softwares, por meio do método de simulação da INI-C: modelagem da edificação no ScketchUp e no OpenStudio, caracterização e simulação no EnergyPlus; modelagem, caracterização e simulação da edificação no DesignBuilder; e utilização do projeto em Revit para caracterização e simulação no DesignBuilder, após processo de interoperabilidade.

Para cada combinação de softwares, foram obtidos os consumos energéticos anuais, as cargas térmicas de resfriamento anuais e as respectivas ENCE Geral de Projeto. Para isso, foram elaboradas modelagens da edificação militar na condição real (tal como no projeto) e na condição de referência (com características estabelecidas pela INI-C, equivalentes à uma edificação com ENCE Classe 'D').

O consumo energético anual da edificação na condição real foi de $32.140,73$ kWh para a combinação utilizando EnergyPlus; $32.457,75$ kWh para a combinação utilizando apenas DesignBuilder; e 32.131,83 kWh para a combinação utilizando Revit e DesignBuilder. Os desvios entre as modelagens foram de no máximo $1,00 \%$, demonstrando boa correlação. Para a modelagem na condição de referência, os consumos energéticos anuais foram, respectivamente, 80.113,46 kWh; 80.112,15 kWh; e 80.120,53 kWh; os desvios entre cada combinação na condição de referência foram de no máximo 0,01\%, com correlação ainda melhor, uma vez que a modelagem da edificação de referência apresenta maior homogeneidade dos elementos construtivos. Os percentuais de economia de energia da edificação real em relação a edificação de referência foram de, respectivamente, $59,88 \% ; 59,49 \%$ e $59,90 \%$. Assim, para todas as combinações de softwares foi obtido ENCE Geral de Projeto Classe 'A'.

A combinação de maior facilidade e rapidez de aplicação foi a do Revit interoperável com DesignBuilder (em média 39h), seguida da modelagem e simulação no próprio DesignBuilder (em média 52h), e por fim, da modelagem em ScketchUp e OpenStudio com simulação no EnergyPlus (em média 70h). Porém, 
cabe ressaltar que é muito importante que o projeto elaborado no Revit passe por simplificações (apresentadas no trabalho) antes de ser exportado para o DesignBuilder, por meio da utilização de um Modelo Analítico de Energia intermediário.

Os resultados desse artigo mostram que a utilização de projeto elaborado em BIM, em conjunto com software de simulação interoperável, agiliza o processo de análise termoenergética de edificações. Concluise que é viável, além de ser vantajosa, a utilização da interoperabilidade entre Revit e DesignBuilder para etiquetagem energética de edificações do Exército Brasileiro, atendendo as diretrizes nacionais, economizando recursos e melhorando o conforto dos usuários dos empreendimentos militares.

\section{REFERÊNCIAS}

ABNT. Associação Brasileira de Normas Técnicas. NBR 15575: Edificações Habitacionais: Desempenho Parte 1 : Requisitos gerais. Rio de Janeiro: ABNT, 2013.

ANDRADE, M. L. V. X.; RUSCHEL, R. C.. Interoperabilidade de aplicativos BIM usados em arquitetura por meio do formato IFC. Gestão \& Tecnologia de Projetos, v.4, n.2, p.76-111, 2009. DOI: http://doi.org/10.4237/gtp.v4i2.102

BAVARESCO, M. V.; MAZZAFERRO, L.; MELO, A. P.; LAMBERTS, R.. Análise Da Precisão De Um Metamodelo Para a Avaliação De Eficiência Energética Em Edificações. In:ENCONTRO NACIONAL DE CONFORTO NO AMBIENTE CONSTRUÍDO, 14. Anais. Balneário Camboriu: ENCAC, 2017.

BRASIL. Decreto n. 10.306, de 2 de abril de 2020. Estabelece a utilização do Building Information Modelling na execução direta ou indireta de obras e serviços de engenharia realizada pelos órgãos e pelas entidades da administração pública federal, no âmbito da Estratégia Nacional de Disseminação do Building Information Modelling: Estratégia BIM BR, instituída pelo Decreto no 9.983, de 22 de agosto de 2019. Brasília: DOU, 2020.

CB3E. Centro Brasileiro de Eficiência Energética de Edificações. Proposta de método para avaliação de eficiência energética de edificações comerciais, de serviços e públicas: Apresentação. CB3E, 2017.

CB3E. Centro Brasileiro de Eficiência Energética de Edificações. Metamodelo online baseado em RNA para cálculo da carga térmica anual de uma edificação. CB3E, 2019.

$\mathrm{CO} / 3^{\circ} \mathrm{GPT}$ E. Comissão de Obras do $3^{\circ}$ Grupamento de Engenharia. Planta de Lay-Out (planta humanizada), estudo de insolação e perspectivas: Construção da Companhia de Comando e Apoio (CCAp). Campo Grande: Diretoria de Obras Militares do Exército Brasileiro, 2017.

EVANS, M.; SHUI, B.. Country report on building energy codes in japan. Washington: Department of Energy, 2009.

FOSSATI, M.; SCALCO, V. A.; LINCZUK, V. C. C.; LAMBERTS, R.. Building energy efficiency: An overview of the Brazilian residential labeling scheme. Renewable and Sustainable Energy Reviews, v.65, p.1216-1231, 2016. DOI: https://doi.org/10.1016/i.rser.2016.06.048

GOUVEIA, A. C. A.; ANDRADE, L. S.; ALVIM, L. G.; MAGALHÃES, A. G.. Obtenção de coordenadas da geometria de um edificação para arquivo do EnergyPlus utilizando o Revit. Revista Construindo, Belo Horizonte, v.6, n.2, 2014.

HENSEN, J. L. M.; LAMBERTS, R.. Building performance simulation for design and operation. Nova lorque: Spon Press, 2011.

IEA. International Energy Agency. Global Status Report for Buildings and Construction 2019. Paris: IEA, 2019a.

IEA. International Energy Agency. Word energy balances: overview. Paris: IAE, 2019b.

INMETRO. Instituto Nacional de Metrologia, Qualidade e Tecnologia. Portaria n. 17, de 16 de janeiro de 2012. Aprova a retificação do RTQ-C: Regulamento Técnico da Qualidade para o Nível de Eficiência Energética de Edificações Comerciais, de Serviços e Públicas. Rio de Janeiro: INMETRO, 2012.

INMETRO. Instituto Nacional de Metrologia, Qualidade e Tecnologia. Manual para Aplicação do RTQ-C. Rio de Janeiro: INMETRO, 2016.

INMETRO. Instituto Nacional de Metrologia, Qualidade e Tecnologia. Portaria n. 248, de 10 de julho de 2018. Consulta Pública da INI-C: Instrução Normativa INMETRO para a Classe de Eficiência Energética de Edifícios Comerciais, de Serviços e Públicos. Aperfeiçoamento do RTQ-C: Regulamento Técnico da Qualidade para a Classe de Eficiência Energética de Edifícios Comerciais, de Serviços e Públicos. Rio de Janeiro: INMETRO, 2018.

KAMEL, E.; MEMARI, A. M.. Review of BIM's application in energy simulation: Tools, issues, and solutions. Automation in Construction, v.97, p.164-180, 2019. DOI: https://doi.org/10.1016/j.autcon.2018.11.008

MELO, A. P.; CÓSTOLA, D.; LAMBERTS, R.; HENSEN, J. L. M.. Assessing the accuracy of a simplified building energy simulation model using BESTEST: The case study of Brazilian regulation. Energy and Buildings, v.45, p.219-228, 2012. DOI: https://doi.org/10.1016/j.enbuild.2011.11.007

MELO, A. P.; CÓSTOLA, D.; LAMBERTS, R.; HENSEN, J. L. M.. Desenvolvimento de um método para aprimorar a precisão do método prescritivo da etiquetagem PROCEL/INMETRO para edifícios comerciais. In: ENCONTRO NACIONAL DE CONFORTO NO AMBIENTE CONSTRUÍDO, 12. Anais. Brasília: ENCAC, 2013.

MPOG. Ministério do Planejamento, Orçamento e Gestão. Instrução Normativa n. 2, de 4 de junho de 2014. Dispõe sobre regras para a aquisição ou locação de máquinas e aparelhos consumidores de energia pela Administração Pública Federal direta, autárquica e fundacional, e uso da Etiqueta Nacional de Conservação de Energia (ENCE) nos projetos e respectivas edificações públicas federais novas ou que recebam retrofit. Brasília: SLTI-MPOG, 2014. 
PIMENTEL, B. P.; BARBOSA, A. T. R.; SOUZA, M. D.. Análise De Métodos De Integração Entre Bim E Simulação Termo Energética De Edificações Militares. Revista Gestão \& Sustentabilidade Ambiental, v.9, p.125, 2020. DOI: http://dx.doi.org/10.19177/rgsa.v9e02020125-146

RACHID, M. N.. Implementação da eficiência energética em uma edificação militar do exército brasileiro. Dissertação (Mestrado em Eficiência Energética e Sustentabilidade) Universidade Federal do Mato Grosso do Sul, Campo Grande, 2018.

RORIZ, M.. Classificação de climas do Brasil: versão 3.0. São Carlos: Associação Nacional de Tecnologia do Ambiente Construído, 2014.

SILVA, P.. Aplicação do programa EnergyPlus como ferramenta do projecto de comportamento térmico de edifícios de habitação. Dissertação (Mestrado em
Engenharia Civil) - Universidade do Porto, Porto, 2010.

TEIXEIRA, A. C.. Processo de etiquetagem de projetos de obras militares para eficiência energética e certificação procel utilizando modelagem de informação da construção. Dissertação (Mestrado em Engenharia de Defesa) - Instituto Militar de Engenharia, Rio de Janeiro, 2018.

VERSAGE, R. S.. Metamodelo para estimar a carga térmica de edificações condicionadas artificialmente. Tese (Doutorado em Engenharia Civil) - Universidade Federal de Santa Catarina, Florianópolis, 2015.

YAMAKAWA, M. A.; WESTPHAL, F. S.. Influência do percentual de abertura nas fachadas e do fator solar dos vidros na etiquetagem do Procel/Inmetro: método prescritivo $x$ simulação. In: ENCONTRO NACIONAL DE CONFORTO NO AMBIENTE CONSTRUÍDO, 11. Anais. Búzios: ENCAC, 2011.

A CBPC - Companhia Brasileira de Produção Científica (CNPJ: 11.221.422/0001-03) detém os direitos materiais desta publicação. Os direitos referem-se à publicação do trabalho em qualquer parte do mundo, incluindo os direitos às renovações, expansões e disseminações da contribuição, bem como outros direitos subsidiários. Todos os trabalhos publicados eletronicamente poderão posteriormente ser publicados em coletâneas impressas sob coordenação da Sustenere Publishing, da Companhia Brasileira de Produção Científica e seus parceiros autorizados. Os (as) autores (as) preservam os direitos autorais, mas não têm permissão para a publicação da contribuição em outro meio, impresso ou digital, em português ou em tradução. 